\title{
Referees for Volume 56/2009
}

The Editors would like to thank the following scholars, who have contributed as referees during 2009.

Giacomo Degli Antoni
Leonardo Becchetti
Enrico Bellino
Stefania Bernabè
Cosimo Beverelli
Ennio Bilancini
Giancarlo Blangiardo
Laura Ferrari Bravo
Stefano Brusoni
Roberta Capello
Floriana Cerniglia
Andrea Colciago
Emilio Colombo
Fedele De Novellis
Enzo Dia
Sheila Dow
Federico Etro
Evelyn Forget
Valeria Gattai
Mario Roberto Gilli
Luciano G. Greco
Michele Grillo

Giacomo Degli Antoni

Leonardo Becchetti

Stefania Bernabè

Cosimo Beverelli

Ennio Bilancini

Giancarlo Blangiard

Stefano Brusoni

Roberta Capello

Floriana Cerniglia

Andrea Colciago

Emilio Colombo

Enzo Dia

Sheila Dow

Michele Grillo

\author{
Harald Hagemann \\ Massimiliano Marcellino \\ Graziella Marzi \\ Giuseppe Micheli \\ Alessandro Missale \\ Ahmad Naimzada \\ Giorgio Barba Navaretti \\ Antonio Nicita \\ Luigi Orsenigo \\ Gianmarco Ottaviano \\ Stefania Ottone \\ Laura Pagani \\ Elisa Portale \\ Michele Ruta \\ Barbara Sena \\ Lucia Tajoli \\ Piero Tedeschi \\ Chiara Tomasi \\ Frederic Tournemaine \\ Edward Tower \\ Stefano Zamagni
}

